\title{
Electron delocalization in amorphous carbon by ion implantation
}

\author{
R. U. A. Khan,* J. D. Carey, and S. R. P. Silva \\ School of Electronic Engineering, I.T. and Mathematics, University of Surrey, Guildford, Surrey GU2 7XH, United Kingdom \\ B. J. Jones and R. C. Barklie \\ Department of Physics, Trinity College, Dublin 2, Ireland \\ (Received 25 September 2000; published 5 March 2001)
}

\begin{abstract}
The electrical properties of amorphous carbon are governed by the high localization of the $s p^{2} \pi$ states, and conventional methods of altering the $s p^{2}$ content result in macroscopic graphitization. By using ion beams we have achieved a delocalization of the $\pi$ states by introducing nanoclustering and hence improving the connectivity between existing clusters, as demonstrated by the increase in the conductivity by two orders of magnitude without modification of the band gap. At higher doses, paramagnetic relaxation-time measurements indicate that exchange effects are present. This unveils the possibility of amorphous carbon-based electronics by tailoring the ion-beam conditions, which we demonstrate in the form of a rectifying device.
\end{abstract}

DOI: 10.1103/PhysRevB.63.121201

PACS number(s): 72.15.- $-\mathrm{v}$

Amorphous carbon $(a-\mathrm{C})$ and hydrogenated amorphous carbon $(a-\mathrm{C}: \mathrm{H})$ consist of a mix of $s p^{2}$ and $s p^{3}$ hybridized phases. By adjusting their ratios a wide range of optical, tribological, and electronic properties are possible. ${ }^{1-3}$ Early calculations by Robertson and O'Reilly ${ }^{4}$ showed that the optical properties of $a-\mathrm{C}$ and $a-\mathrm{C}: \mathrm{H}$ are ruled by the size of the $s p^{2}$ clusters, which can either be in the form of aromatic rings or olefinic chains. These clusters may introduce states at or near the Fermi level, depending on their size and on whether they are odd or even numbered. ${ }^{5}$ Hopping between clusters has been proposed ${ }^{6}$ as a possible mechanism for room-temperature conduction. Recent calculations ${ }^{7}$ showed that the $s p^{2}$ states remain localized even up to $80 \% s p^{2}$ bonding, well above the percolation threshold. This localization of states has been attributed to dihedral bond angle disorder, and such localized states result in hopping with a low activation energy at room and low temperatures, and bandtail conduction at higher temperatures. ${ }^{8,9}$

Conventional techniques to alter the $s p^{2}$ content for $a-\mathrm{C}: \mathrm{H}$ films are by varying the deposition parameters such as dc self-bias, ${ }^{10}$ through the addition of $\mathrm{N}^{11}$ or through thermal annealing. ${ }^{12}$ Conventional annealing results in a macroscopic $s p^{2}$ restructuring of the film, and the formation of larger and larger $s p^{2}$ clusters. An alternative approach is to perform localized heating by means of ion implantation, whereby it is possible to deposit highly localized energy in a controlled manner. Previous studies of damage as a result of ion implantation found extensive $s p^{2}$ restructuring (graphitization) at very high doses, ${ }^{13,14}$ and a consequent increase in film conductivity. However, by controlling the ion dose we show that it is possible to enhance the conductivity through the film, but prevent macroscopic graphitization. This results in an effective delocalization of electrons within the film.

Films were grown on $\mathrm{Si}$ and Corning 7059 substrates on the water-cooled earthed electrode of a rf plasma-enhanced chemical-vapor deposition system described elsewhere. ${ }^{15}$ Films were implanted with B ions at energies of 20 and 26 $\mathrm{keV}$ in the dose range of $2 \times 10^{12}-2 \times 10^{16} \mathrm{~cm}^{-2}$, and were simulated using SUSPRE $v 1.4$ assuming a $\mathrm{H}: \mathrm{C}$ ratio of $1: 1$ and a film density of $1.72 \mathrm{~g} \mathrm{~cm}^{-3}$. The projected ranges were found to be 60 and $76 \mathrm{~nm}$ for the two implant energies, with a straggle of $13 \mathrm{~nm}$ in each case. Electrical measurements were made on $\mathrm{Au} / a-\mathrm{C}: \mathrm{H} / \mathrm{Cr}$ device structures. Roomtemperature electron paramagnetic resonance measurements were made using a $\mathrm{TM}_{011}$ mode cavity at about $9.9 \mathrm{GHz}$, and a field modulation of $100 \mathrm{kHz}$. Spin concentrations were determined by comparison with a Varian standard sample of pitch in $\mathrm{KCl} ; g$ values were determined by comparing the line position to that of the resonance from the $\mathrm{F}^{+}$center in $\mathrm{MgO}$. Relaxation times were measured using the power saturation method discussed elsewhere. ${ }^{16}$

The variation in optical band gap and resistivity of the $a-\mathrm{C}: \mathrm{H}$ films is shown in Fig. 1. On increasing the dose to $2 \times 10^{14} \mathrm{~cm}^{-2}$, the Tauc gap remains approximately constant

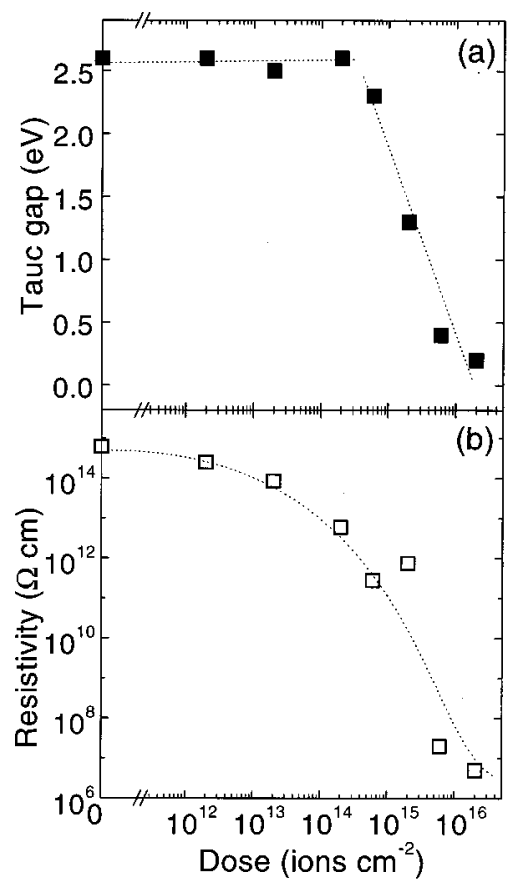

FIG. 1. Variation in the (a) Tauc optical band gap and (b) resistivity as a function of boron ion dose. 
within error, but there is a decrease in resistivity of two orders of magnitude. By contrast, for doses greater than 2 $\times 10^{14} \mathrm{~cm}^{-2}$, the optical gap falls and eventually reaches 0.2 $\mathrm{eV}$ at the highest ion dose, with the film resistivity decreasing from $6 \times 10^{12}$ to $5 \times 10^{6} \Omega \mathrm{cm}-$ a decrease by nearly six orders of magnitude. The $\mathrm{H}$ content falls from 55 to 25 at. \% over the dose range, as measured using elastic recoil detection analysis.

Electron paramagnetic resonance (EPR) measurements were made on all implanted films; however, signals from C-related centers with $g \sim 2.0028$ are only observed for films implanted with doses higher than $2 \times 10^{15} \mathrm{~cm}^{-2}$. At lower doses the EPR signal is dominated by signals with $g$ $\sim 2.0055-2.0061$, associated with Si-related defects Figure 2(a) shows that the spin concentration increases with dose from $3 \times 10^{17} \mathrm{~cm}^{-3}$ in the unimplanted sample, up to 2.7 $\times 10^{20} \mathrm{~cm}^{-3}$ at the highest dose of $2 \times 10^{16} \mathrm{~cm}^{-2}$. In this dose range the EPR signal has a Lorentzian line shape, and the peak-to-peak linewidth $\Delta B_{\mathrm{pp}}$ reduces from 0.83 to 0.13 $\mathrm{mT}$, as shown in Fig. 2(b). The variation in the spin lattice, $T_{1}$, and the spin-spin, $T_{2}$, relaxation time with dose are shown in Fig. 2(c). As the implantation dose is increased, $T_{1}$ shortens from $1.1 \times 10^{-5}$ to $6 \times 10^{-8} \mathrm{~s}$, while $T_{2}$ is observe to lengthen from $1.4 \times 10^{-8}$ to $5.1 \times 10^{-8} \mathrm{~s}$. Using the method employed in Ref. 17, we have calculated the dipolar $\left(\omega_{p}\right)$ and exchange $\left(\omega_{c}\right)$ frequencies from the available values of $T_{1}$ and $T_{2}$. The ratio of $\omega_{c} / \omega_{p}$ is found to be 1.9 for the unimplanted sample, which increases to 4.8 for the film implanted to $2 \times 10^{15} \mathrm{~cm}^{-2}$, and then rises to 16.7 and 50 for the two highest doses. The ratio of $\omega_{c} / \omega_{p}$ indicates the efficiency in which the rapidly fluctuating magnetic field is able to average out the static dipolar broadening. Values over 10 indicate an efficient motional narrowing, in this case as a result of extensive $s p^{2}$ reordering. This occurs only in the two highest doses. In the case of the $2 \times 10^{15} \mathrm{~cm}^{-2}$ sample, extensive $s p^{2}$ reordering has not occurred at this dose, but motional narrowing has increased the ratio as compared to its unimplanted value.

These results indicate that there exist two regimes; one for doses up to and including $2 \times 10^{14} \mathrm{~cm}^{-2}$, where the Tauc gap remains constant and the film resistivity falls by about two orders of magnitude; and another regime above this dose where, by the highest dose of $2 \times 10^{16} \mathrm{~cm}^{-2}$, there is a near closure of the optical gap, a further reduction of the film resistivity by six orders of magnitude, and where exchange effects dominate the EPR linewidth and relaxation times. In order to analyze the resistivity variation, the equations for variable-range hopping $(\mathrm{VRH})$ for electrons at the Fermi level, originally derived by Mott and Davis, ${ }^{18}$ have been applied to these films. ${ }^{14}$ Using a localization factor $\alpha$, of 7.5 $\times 10^{6} \mathrm{~cm}^{-1},{ }^{19}$ the required density of states at the Fermi level, $N\left(E_{F}\right)$, would have to exceed $10^{29} \mathrm{~cm}^{-3} \mathrm{eV}^{-1}$. From the measured spin density of $3 \times 10^{20} \mathrm{~cm}^{-3}$ at the highest dose, an estimate of $N\left(E_{F}\right)$ can be obtained by assuming a maximum correlation energy of $2 \mathrm{eV} .{ }^{20}$ This gives $N\left(E_{F}\right)$ $\sim 10^{20} \mathrm{~cm}^{-3} \mathrm{eV}^{-1}$, considerably less than that required for VRH. In the model of Ref. 6, conduction at room temperature occurs via hopping between neighboring $s p^{2}$ islands,

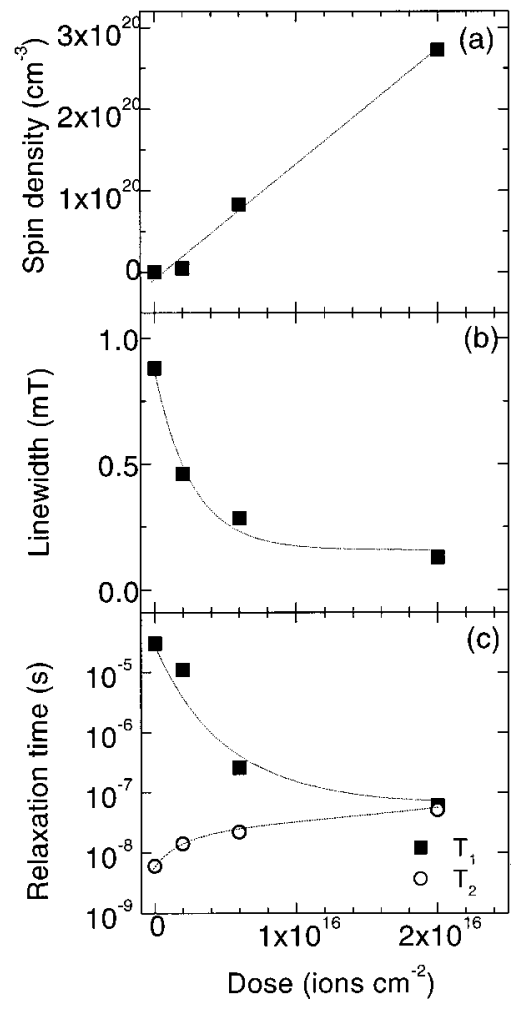

FIG. 2. Variation of (a) the C-related spin density, (b) peak-topeak linewidth, and (c) relaxation times as a function of boron ion dose.

and so is determined by the concentration of gap states and the separation between the $s p^{2}$ islands, as shown schematically in Fig. 3. As the $s p^{2}$ content of the as-deposited films is likely to be low (30\%), the hopping distance is likely to be large, resulting in low conductivity. During ion implantation, localized heating would result in the formation of highly localized $s p^{2}$ sites, which are small if the ion dose is below the graphitization threshold, at which the ion tracks start to coincide as in the case of diamond. ${ }^{21}$ It is likely that the concentration of these introduced sites is proportional to the ion dose. Implantation at low doses introduces nanoclustering below the average cluster size, which maintains the wide
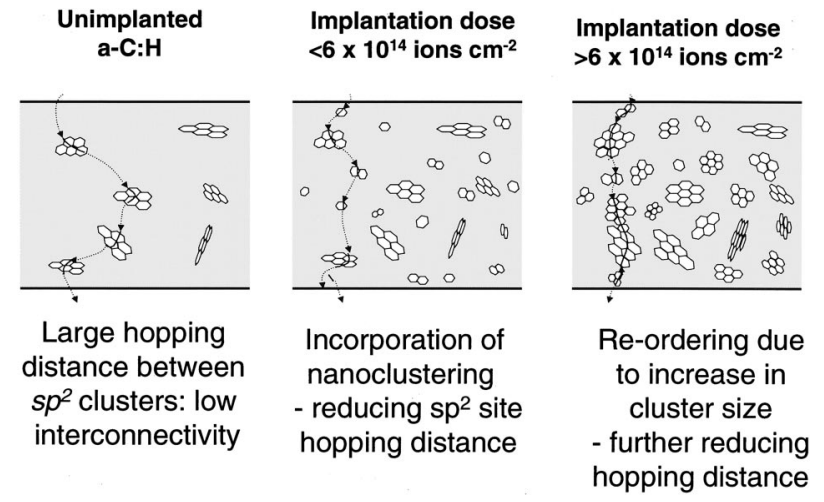

FIG. 3. Proposed effect of ion implantation on hopping conductivity between adjacent clusters, below and above the dose of 6 $\times 10^{14} \mathrm{~cm}^{-2}$. 


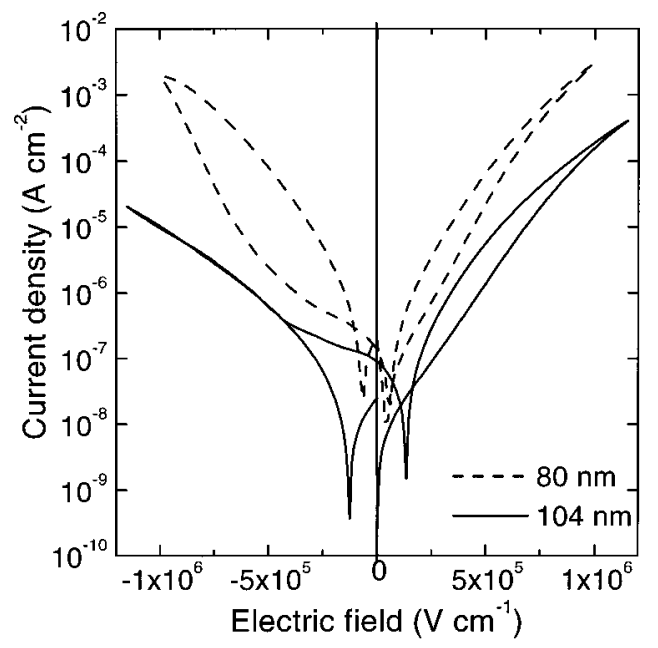

FIG. 4. Current density vs applied electric field characteristics for two $a-\mathrm{C}: \mathrm{H}$ films implanted to a dose of $2 \times 10^{15}$ ions $\mathrm{cm}^{-2}$ of 80 and 104-nm thickness. Simulations predict that the B ions have penetrated all the way through the thinner film but only partially through the thicker film.

band gap but increases the conductivity by decreasing the hopping distance. At higher doses, the film restructures over the bulk of the film, the band gap collapses accordingly, and the effects of exchange become prevalent.

Reductions in film resistivity with concomitant falls in the optical band gap as a function of ion dose were reported in ta-C by McCulloch et al., ${ }^{13}$ and in diamondlike $a-\mathrm{C}: \mathrm{H}$ by Prawer et al. ${ }^{14}$ In the former, the reduction of the optical gap and the decrease in film resistivity occurred even at the lowest doses investigated $\left(10^{13} \mathrm{~cm}^{-2}\right)$, and appeared to be concomitant. In the latter study, the resistivity remained constant up to a dose of $10^{15} \mathrm{~cm}^{-2}$ and then decreased, and the optical gap decreased from about $1.5 \mathrm{eV}$ in the as-grown film to less than $1 \mathrm{eV}\left(2 \times 10^{17} \mathrm{~cm}^{-2}\right)$, following the variation in the resistivity. It is interesting to note that the $\mathrm{H}$ concentration in the $a-\mathrm{C}: \mathrm{H}$ films decreased from 35 at. \% (as grown) to less than 3 at. $\%$ at the highest dose. So, in both cases, as the optical band gap falls there is a concomitant fall in resistivity.

Since the film implanted at $2 \times 10^{15} \mathrm{~cm}^{-2}$ may exhibit evidence of exchange but still possesses a band gap of $1.3 \mathrm{eV}$, this film has favorable properties which may be applicable for device applications. Below a dose of $2 \times 10^{14} \mathrm{~cm}^{-2}$, the conduction mechanism is space charge limited, ${ }^{22}$ as inferred from the thickness scaling law $\left(J \propto V^{2} / d^{3}\right)$. Above a dose of $6 \times 10^{15} \mathrm{~cm}^{-2}$, a linear $\ln J / E$ versus $E^{1 / 2}$ relationship is obtained, consistent with Poole-Frenkel conduction. The film implanted at a dose of $2 \times 10^{15} \mathrm{~cm}^{-2}$ shows a voltagedependent current enhancement, as shown in Fig. 4, which does not follow either of the above mechanisms. We investigated the $J$ versus $E$ characteristics of two films implanted at the same dose with film thicknesses of 80 and $104 \mathrm{~nm}$. In the case of the $80 \mathrm{~nm}$ thick film, the projected ranges derived from SUSPRE indicate that the film would be implanted throughout the whole of its thickness, while the 104-nmthick film is likely to have an unimplanted region close to the substrate. The former sample shows switching behavior in both forward and reverse voltage directions, while the latter shows switching in the forward direction only, i.e., rectification of over one order of magnitude. We have applied a Schottky barrier-lowering mechanism for this film using the equation $^{23}$

$$
J=A^{* *} T^{2} \exp \left[\frac{q \Phi}{k T}+\frac{\beta}{2} \sqrt{E}\right]
$$

where

$$
\beta=\frac{1}{k T} \sqrt{\left(\frac{e^{3}}{\pi \epsilon_{r} \epsilon_{0}}\right)^{1 / 2}},
$$

where $A^{* *}$ is Richardson's constant, $k$ is Boltzmann's constant, $T$ is the absolute temperature, $e$ is $1.6 \times 10^{-19} \mathrm{C}, \epsilon_{0}$ is the permittivity of free space, and $\epsilon_{r}$ is the relative permittivity. From the slope of the $\ln J$ versus $E^{1 / 2}$ characteristic, a value of 2.4 is obtained for $\epsilon_{r}$, and an approximate barrier height of $0.8 \mathrm{eV}$ is calculated. Since the 104-nm-thick film is likely to be partially implanted and the 80 -nm-thick film is likely to be completely implanted, there must be a dependence on the back contact which determines whether the current enhancement occurs in the negative voltage region or not. This can be explained if a contact which has received a suitable dose of ions is able to trap charge. This trapping process, as a result of the applied voltage, would then result in a space-charge region close to the contact, resulting in a narrowing of the electrical thickness of the film and hence a modification of the barrier height at the contact on the other side of the film. If a film is only partially implanted, then this trapping process can only occur at the surface contact. This would mean that current enhancement would only occur in the positive voltage segment and not the negative voltage segment. In this way, a barrier-limiting process can account for the conduction properties of these films. This ion-beaminduced barrier is as a consequence of the controlled increase in the bulk conductivity or delocalization of bulk states, previously not possible in our films.

The characteristics of the rectifying diode demonstrated here may be compared to the Schottky diodes reported in Ref. 24, the $p-n$ junction diode reported in Ref. 25, and the $a-\mathrm{C}: \mathrm{H}$ and $t a-\mathrm{C}$ heterojunction diodes reported in Refs. 26 and 27. In the case of the Schottky diode, point contacts were required which may have reduced the efficiency of carrier injection from the top contact, and for $p-n$ junction diode, the forward to reverse rectification ratio was only about six. For the $a-\mathrm{C}: \mathrm{H}$-based heterojunction diode ${ }^{26}$ the rectification ratio was two orders of magnitude. However, before electrical measurements were performed a potential of $200 \mathrm{~V}$ was applied to the device in order to measure any current gain with applied voltage, and its effects on both the film microstructure and the contacts are not known at present. No such voltage was applied in the case of the ion-implanted diodes which were fabricated in this study.

To conclude, we have implanted hydrogenated amorphous carbon films, and observed evidence of a controllable increase in conductivity, as a function of ion dose. This is only possible using ion implantation, as by using this method 
one can introduce localized heating in order to promote nanoclustering. The high-field conduction properties of the material appear to show a contact-limited current at intermediate ion doses, which allows us to suggest that devices using the enhanced conduction properties of the material are possible. We have demonstrated that it is possible to create barrier-controlled devices in films with partially delocalized electrons which still possess an appreciable band gap.
*Electronic address: r.khan@eim.surrey.ac.uk

${ }^{1}$ C. A. Davis, S. R. P. Silva, R. E. Dunin-Borkowski, G. A. J. Amaratunga, K. M. Knowles, and W. M. Stobbs, Phys. Rev. Lett. 75, 4258 (1995).

${ }^{2}$ D. R. McKenzie, D. Muller, and B. A. Pailthorpe, Phys. Rev. Lett. 67, 773 (1991).

${ }^{3}$ Y. Lifshitz, S. R. Kasi, J. W. Rabalais, and W. Eckstein, Phys. Rev. B 41, 10468 (1990).

${ }^{4}$ J. Robertson and E. P. O'Reilly, Phys. Rev. B 35, 2946 (1987).

${ }^{5}$ J. Robertson, Adv. Phys. 35, 317 (1986).

${ }^{6}$ D. Dasgupta, F. Demichelis, and A. Tagiaferro, Philos. Mag. B 63, 1255 (1991).

${ }^{7}$ C. W. Chen and J. Robertson, J. Non-Cryst. Solids 227, 602 (1998).

${ }^{8}$ R. Orzeszko, W. Bala, K. Fabisiak, and F. Rozploch, Phys. Status Solidi A 81, 579 (1984).

${ }^{9}$ A. Helmbold, P. Hammer, J. U. Thiele, K. Rohwer, and D. Meissner, Philos. Mag. B 72, 335 (1995).

${ }^{10}$ Y. Lifshitz, S. R. Kasi, and J. W. Rabalais, Phys. Rev. Lett. 62, 1290 (1989).

${ }^{11}$ S. R. P. Silva, J. Robertson, G. A. J. Amaratunga, B. Rafferty, L. M. Brown, J. Schwan, D. F. Franceschini, and G. Mariotto, J. Appl. Phys. 81, 2626 (1997).

${ }^{12}$ Y. Bounouh, M. L. Theye, A. Dehbi-Alaoui, A. Matthews, and J. P. Stoquet, Phys. Rev. B 51, 9597 (1995).

${ }^{13}$ D. G. McCulloch, E. G. Gerstner, D. R. McKenzie, S. Prawer, and R. Kalish, Phys. Rev. B 52, 850 (1995).

${ }^{14}$ S. Prawer, R. Kalish, M. E. Adel, and V. Richter, J. Appl. Phys. 61, 4492 (1987).

${ }^{15}$ J. V. Anguita, S. R. P. Silva, A. P. Burden, B. J. Sealy, S. Haq, M. Hebron, I. Sturland, and A. Prichard, J. Appl. Phys. 86, 6276 (1999).

${ }^{16}$ C. P. Poole, Electron Spin Resonance (Wiley, New York, 1983), p. 476.

${ }^{17}$ R. C. Barklie, M. Collins, and S. R. P. Silva, Phys. Rev. B 61, 3546 (2000).

${ }^{18}$ N. F. Mott and E. A. Davis, Electronic Processes in NonCrystalline Materials (Oxford University Press, 1971), Chap. 1.

${ }^{19}$ R. M. Hill, Philos. Mag. 23(181), 59 (1971).

${ }^{20}$ J. Ristein, J. Schäfer, and L. Ley, Diamond Relat. Mater. 4, 509 (1995).

${ }^{21}$ S. Prawer and R. Kalish, Phys. Rev. B 51, 15711 (1995).

${ }^{22}$ A. Rose, Phys. Rev. 97, 1538 (1955).

${ }^{23}$ P. Mark and T. E. Hartman, J. Appl. Phys. 39, 2163 (1968).

${ }^{24}$ S. Paul and F. J. Clough, Diamond Relat. Mater. 7, 1734 (1998).

${ }^{25}$ D. R. McKenzie, Y. Yin, C. A. Davis, B. A. Pailthorpe, G. Amaratunga, and V. S. Veerasamy, Diamond Relat. Mater. 3, 353 (1994).

${ }^{26}$ G. A. J. Amaratunga, W. I. Milne, and A. Putnis, IEEE Electron Device Lett. 11, 33 (1990).

${ }^{27}$ G. A. J. Amaratunga, D. E. Segal, and D. R. McKenzie, Appl. Phys. Lett. 59, 69 (1991). 